\title{
Genre et nombre des emprunts nominaux de l'arabe marocain au français : étude phonétique et morphologique
}

Gender and number of nominal borrowings of Moroccan Arabic from French: phonetic and morphological study

\section{Mjid El garni}

\section{OpenEdition}

Journals

Édition électronique

URL : https://journals.openedition.org/pratiques/10897

DOI : 10.4000/pratiques. 10897

ISSN : 2425-2042

\section{Éditeur}

Centre de recherche sur les médiations (CREM)

\section{Référence électronique}

Mjid El garni, « Genre et nombre des emprunts nominaux de l'arabe marocain au français : étude phonétique et morphologique », Pratiques [En ligne], 191-192 | 2021, mis en ligne le 15 décembre 2021, consulté le 03 janvier 2022. URL : http://journals.openedition.org/pratiques/10897 ; DOI : https:// doi.org/10.4000/pratiques. 10897

Ce document a été généré automatiquement le 3 janvier 2022

(c) Tous droits réservés 


\section{Genre et nombre des emprunts nominaux de l'arabe marocain au français : étude phonétique et morphologique}

Gender and number of nominal borrowings of Moroccan Arabic from French: phonetic and morphological study

\section{Mjid El garni}

\section{1- Introduction}

1 L'emprunt lexical est considéré comme une source indispensable pour l'enrichissement des langues. C'est l'un des mécanismes naturels de l'évolution linguistique (J. Hamers, 1997 ; C. Loubier, 2011). En d'autres termes, c'est une forme de néologie que toutes les langues utilisent pour continuer à exister, à ce propos B. Quémada affirme qu' : "Une langue qui ne connaitrait aucune forme de néologie serait déjà une langue morte, et l'on ne saurait contester que l'histoire de toutes nos langues n'est, en somme, que l'histoire de leur néologie » (Quémada, 1971, p. 37). C. Hagège voit même en l'emprunt un « facteur constitutif de la vie des langues, laquelle est liée à celle des populations mêmes (sic) qui les parlent, et il n'existe pas de langue qui n'ait à tel ou tel moment, fait des emprunts à d'autres ». C. Hagège (2006, p. 42). L'emprunt linguistique est donc un phénomène très ancien, mais aujourd'hui il tend à s'amplifier davantage par le biais des médias et notamment l'Internet. La quantité et la nature des emprunts lexicaux introduits dans une langue dépendent des rapports historiques, économiques et politiques qu'entretiennent les pays.

2 L'emprunt du français par l'arabe marocain, en plus de ces facteurs, peut être attribué à des raisons sociales, car la langue française jouit d'un certain prestige au Maroc comme étant la langue de l'élite. "La valeur attribuée au mot emprunté est une question 
sociale et nationale ; selon que l'idiome et le peuple auxquels on fait des emprunts sont regardés inférieurs ou supérieurs, ces emprunts descendent ou montent en dignité " (Deroy, 1956, p. 18).

3 Ainsi, les emprunts à une langue valorisée par les locuteurs locaux pénétreront facilement et rapidement leur système linguistique à tel point que leur origine sera même oubliée, tandis que les emprunts à des langues dévalorisées ne parviendront même pas à leur trouver une place dans la langue d'accueil.

4 Par-delà la valeur sociolinguistique de la langue, sa structure consonantique joue également un rôle dans l'acceptation ou le rejet d'emprunt. Ainsi, les langues ayant un système consonantique faible sont plus ou moins imperméables aux emprunts linguistiques, comme c'est le cas par exemple du finnois, qui a une structure consonantique réduite qui ne lui permet pas d'intégrer facilement de nouveaux emprunts.

5 Le grand nombre d'emprunts en arabe marocain, surtout au français, nous a amenés à réaliser cette étude. Le choix de ce thème a également été motivé par la rareté de la littérature traitant de ce sujet. À travers cette modeste étude, nous tenterons de combler, ne serait-ce que partiellement, cette carence.

6 Pour qu'il soit complètement intégré par la langue d'accueil, l'emprunt lexical doit subir des modifications phonologiques et morphosyntaxiques (Haugen, 1950 ; Humbley, 1974 ; Guilbert, 1975). Dans cette étude sur les emprunts lexicaux de l'arabe marocain au français, nous nous sommes centré, à partir d'un corpus écrit issu de dictionnaires et d'une enquête concernant l'usage oral, sur deux des aspects morphosyntaxiques spécifiques de l'intégration des emprunts lexicaux d'origine française, à savoir l'attribution du genre et celle des marques de nombre. La présente étude montre que le comportement des emprunts en termes de genre et de nombre n'est pas standardisé : certains gardent leur genre d'origine, d'autres le perdent au profit de la langue emprunteuse. En ce qui concerne le nombre (pluriel), on constate que les emprunts intègrent en général les règles de formation du nombre de l'arabe marocain. À partir de la description précise de 67 lexèmes, nous discuterons des raisons linguistiques permettant d'expliquer les différents comportements de ces emprunts en termes de genre et de nombre.

7 Concernant la méthode utilisée dans l'analyse de l'intégration de ces mots français, nous nous sommes basés sur trois théories. La première est celle de E. Haugen (1950), qui prévoit deux aspects principaux de l'analyse des emprunts, l'aspect phonologique et l'aspect morphologique. La deuxième théorie est celle de J. Rey-Debove (1973), cette linguiste fait état de trois phases d'intégration des emprunts, la phase métalinguistique, la phase à connotation métalinguistique-autonyme et la phase d'aboutissement marquant l'intégration complète des emprunts dans la langue d'accueil. La dernière théorie retenue comme fondement à notre étude est celle de J. Humbley (1974). Ce dernier propose trois étapes dans l'étude des emprunts : l'identification, la modification et les causes. Selon lui, l'analyse des emprunts linguistiques peut couvrir toutes les dimensions de la langue : lexique, phonologie, morphologie, ainsi que l'orthographe et la sémantique. 


\section{2- L'emprunt linguistique :}

8 L'emprunt linguistique, comme mentionné ci-dessus, a toujours été un sujet de controverse entre linguistes. Certains comme A. Meillet (1921) ne voient en l'emprunt aucune utilité et le considèrent comme "un fait marginal» (Meillet, 1921, cité par Baggioni, 1994, p. 3). Ce point de vue est d'ailleurs partagé par plusieurs linguistes (Du bellay, 1972; Etiemble, 1964 ; De Gourmont, 1988) qui estiment que l'emprunt linguistique est facteur de destruction et d'appauvrissement de la langue d'acueil. D'autres linguistes, en revanche, considèrent l'emprunt linguistique comme un fait indispensable pour l'évolution des langues. ${ }^{1}$

Quelle que soit la façon dont les linguistes considèrent l'emprunt, celui-ci reste un phénomène universe $\mathrm{l}^{2}$ comme le souligne J.-M. Chadelat $(2000$, p. 11$)$ : « quelles que soient en effet leurs techniques, leurs institutions ou leurs façons de vivre, tous les groupes humains empruntent des éléments et des formes issus d'autres systèmes linguistiques que les leurs ».

10 En ce qui concerne la définition de l'emprunt linguistique ${ }^{3}$, nous avons choisi celle donnée par J. Dubois (1973, p. 188) dans son Dictionnaire de Linguistique pour sa clarté et sa pertinence :

Il y a emprunt linguistique quand un parler A utilise et finit par intégrer une unité ou un trait linguistique qui existait précédemment dans un parler B et que A ne possédait pas ; l'unité ou le trait emprunté sont eux-mêmes appelés emprunt.

11 Selon cette définition, on comprend que l'emprunt linguistique n'est pas toujours lexical, c'est-à-dire que la langue emprunteuse peut incorporer dans son système linguistique aussi bien les unités lexicales que tout autre élément de la langue (monème, phonème, élément syntaxique, signification, élément stylistique, etc.). Ce fait est clairement confirmé par L. Deroy $(1956)^{4}$ qui pense que l'emprunt peut prendre des formes variées et affecter tous les niveaux de la langue. Pourtant, notre intérêt est uniquement porté sur la composante lexicale du fait qu'elle est la partie la plus concernée par l'emprunt comme nous le fait remarquer M. Tournier $(1985$, p. 315) : « parmi les emprunts graphiques, phonologiques, morphosyntaxiques et lexicaux, ces derniers sont de très loin les plus fréquents. »

\subsection{L'emprunt lexical}

12 L'emprunt lexical, selon C. Loubier (2011, p. 10), est le "Procédé par lequel les utilisateurs d'une langue adoptent intégralement, ou partiellement, une unité ou un trait linguistique ». Ce type d'emprunt linguistique concerne uniquement les mots dans leur relation forme-sens, et c'est par ce trait qu'il se distingue des autres catégories d'emprunts linguistiques (phonétique et syntaxique), c'est le lexique qui génère le plus d'emprunts (Loubier, 2011).

13 Une autre définition légèrement différente de celle de C. Loubier est donnée par J. Humbley (1974, p. 52) :

L'emprunt lexical au sens strict du terme est le processus par lequel une langue L1 dont le lexique est fini et déterminé dans l'instant $T$, acquiert un mot M2 (expression et contenu) qu'elle n'avait pas et qui appartient au lexique d'une Langue L2 (également fixe et déterminé). 
14 L'emprunt ne se fait donc pas toujours de la même manière, il dépend de la période historique, et d'autres contextes comme le confirme J. Dubois (1980, p. 189) : "L'intégration du mot emprunté à la langue emprunteuse se fait de manières très diverses selon les mots et les circonstances. Ainsi, le même mot étranger, emprunté à des époques différentes, prend des formes variées. »

En effet, il n'y a pas de critères stricts et fermés qui régissent l'intégration des emprunts, rien n'est prévisible, tout dépend des langues emprunteuses et prêteuses à un moment donné.

\section{2- Les types d'emprunts lexicaux}

Tout d'abord, il convient de souligner que les linguistes ne sont pas d'accord sur la typologie des emprunts lexicaux. Nous nous limiterons à la classification d'H. Walter et à celle de C. Loubier. H. Walter (2006, p. 103-105) distingue sept types d'emprunts : « les xénismes, les xénismes "naturalisés", les emprunts purs et simples, les emprunts sémantiquement et grammaticalement modifiés, les pseudoemprunts, les calques, les emprunts provisoires et les emprunts qui réussissent ". Tandis que C. Loubier (2011, p. 15-16) les ramène à quatre : l'emprunt intégral, l'emprunt hybride, le calque et le faut emprunt.

Étant donné que ces quatre types d'emprunt sont les plus connus et les plus récents dans la littérature, il convient de les expliquer, ne serait-ce que de manière concise :

- L'emprunt intégral: c'est la lexie transférée vers la langue réceptrice sans modification aucune aussi bien au niveau du sens qu'au niveau de la forme. C'est le cas, par exemple, du mot « computer » qui a été transféré dans son intégralité (forme et sens) de l'anglais au français.

- L'emprunt hybride: c'est un mot dont on a conservé une partie et substitué l'autre par un élément de la langue emprunteuse. Le mot «halalisation », par exemple, est composé d'une partie arabe [Halal] et d'une partie française (-isation).

- Le calque: l'adoption du sens d'un mot étranger par un mot déjà existant dans la langue réceptrice. Par exemple, le mot composé français "gratte-ciel», qui se calque sur la construction anglaise « skyscraper».

- Le faux emprunt : c'est un mot qu'on prend pour un emprunt intégral, alors qu'il ne l'est pas parce qu'il n'a pas d'équivalent dans la langue donneuse. «Tennisman » par exemple est un mot créé en français par imitation de l'anglais, mais le mot n'existe pas chez les anglophones.

Une catégorisation un peu particulière est celle établie par C. Myers-Scotton (2002) qui distingue deux types d'emprunts : les emprunts culturels et les emprunts de base. Il entend par emprunt culturel la dénomination des objets ou concepts nouvellement introduits à la langue d'accueil, tandis qu'il définit l'emprunt de base comme un terme d'une langue étrangère qui remplace des mots déjà existants dans la langue autochtone.

\section{3- Problématique}

Bien que les linguistes ne soient pas tous d'accord au sujet de l'emprunt, notamment en ce qui concerne sa définition, son utilité, ses types, etc., néanmoins tous s'accordent sur le fait que l'emprunt lexical doit obligatoirement se conformer aux règles 
phonologiques et morphosyntaxiques de la langue emprunteuse pour pouvoir s'y intégrer complètement.

Dans cette contribution, nous allons tenter de répondre à deux questions bien précises :

- Quels sont les changements phonétiques et les modifications morphologiques que subissent les emprunts lexicaux au français pour s'adapter au genre et au nombre de l'arabe marocain.

- Quelles justifications linguistiques peut-on donner à ces changements?

\section{4- Corpus d'étude}

Pour rassembler le corpus, nous avons, dans un premier temps, consulté deux ouvrages lexicographiques de l'arabe marocain, il s'agit du Dictionnaire Colin de l'arabe dialectal marocain (Iraqui Sinaceur, 1994) et Qamus ad-dariza al-mayribiya (Mgherfaoui, 2017). Le corpus oral est constitué de neuf interviews de locuteurs natifs marocains de profils différents (voir le tableau 1). Il a été enregistré sur une durée totale de $3 \mathrm{~h} 06$ minutes. Le nombre total recueilli des emprunts au français s'élève à : 709 dont 589 ont été fournis par les supports écrits et 120 ont été récolté à l'aide des interviews. La question posée aux informateurs était la suivante : comment vous appelez cet objet? Il convient de signaler qu'en plus des enregistrements je me suis appuyé également sur mon intuition en tant que locuteur natif.

\begin{tabular}{|l|l|l|l|l|}
\hline Profils Noms & Sexe & Âge & Statut social & Niveau d'instruction académique \\
\hline Mohammed & M & 47 ans & Électricien d'automobile & 1 A.secondaire \\
\hline Abdslam & M & 39 ans & Mécanicien & $3^{e}$ collège \\
\hline Noureddine & M & 52 ans & Maçon & Illettré \\
\hline Youssra & F & 27 ans & Cuisinière & Bachelier \\
\hline Yassine & M & 41 ans & Marin & Bachelier \\
\hline Ahmed & M & 50 ans & Tailleur & $5^{\text {e } \text { A. primaire }}$ \\
\hline Farid & M & 33 ans & Réparateur de motocycle & Illettré \\
\hline Abderrahim & M & 62 ans & Droguiste & 1.A.collège \\
\hline Fatima & F & 29 ans & Pharmacienne & Licencié \\
\hline
\end{tabular}

Pour la transcription des mots d'emprunts, nous avons choisi l'alphabet phonétique international pour sa capacité à calquer phonétiquement les mots tels qu'ils sont prononcés par les locutrices et les locuteurs. Dans le tableau suivant figurent les 67 emprunts dont nous nous sommes servis dans cette étude : 


\begin{tabular}{|c|c|}
\hline 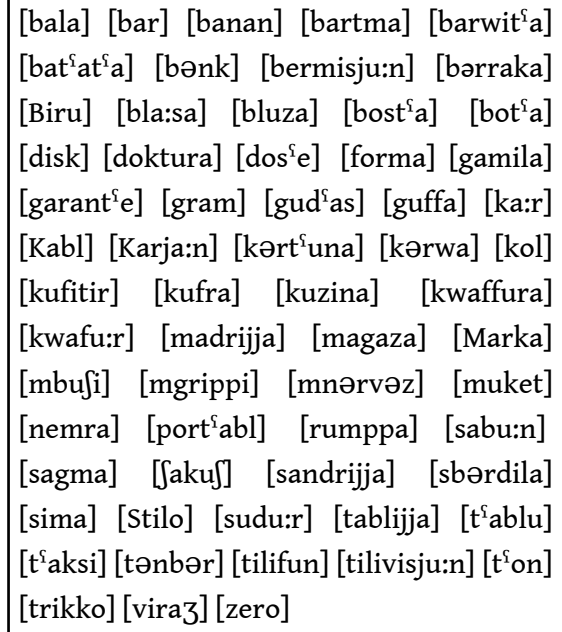 & $\begin{array}{l}\text { Une pelle Un bar Une banane Un appartement Une } \\
\text { brouette Une patate Une banque Une permission Une } \\
\text { baraque Un bureau Une place Une blouse Une poste } \\
\text { Une bouteille Un disque Un doctorat Un dossier Un } \\
\text { format Une gamelle Une garantie Un gramme Une } \\
\text { godasse Un couffin Un car Un câble Une carrière Un } \\
\text { carton Une courroie Un col Une confiture Un coup } \\
\text { franc Une cuisine Une coiffeuse Un coiffeur Un madrier } \\
\text { Un magasin Une marque Bouché Grippé Énervé Une } \\
\text { moquette Un numéro Un portable Un rond-point Un } \\
\text { savon Un segment Une sacoche Un cendrier Une } \\
\text { espadrille Un ciment Un stylo Un soudeur Un tablier } \\
\text { Un tableau Un taxi Un timbre Un téléphone Une } \\
\text { télévision Une tonne Un tricot Un virage Un zéro }\end{array}$ \\
\hline
\end{tabular}

\section{5- Conditions phonétiques et syllabiques de l'attribution du genre aux lexèmes empruntés}

Étant donné la différence entre les deux systèmes linguistiques: français et arabe marocain, l'emprunt lexical au français en plus des modifications phonologiques n'échappe pas à d'autres adaptations à caractère morphosyntaxique. Il s'agit de l'adjonction d'affixes marocains à la base lexicale française, ce phénomène reste un procédé linguistique incontournable pour une intégration grammaticale complète des emprunts lexicaux au français dans le système linguistique de l'arabe marocain.

En matière de genre, nous avons constaté que certains emprunts au français conservent le genre d'origine alors que d'autres acquièrent celui de la langue emprunteuse. La forme phonétique et la structure syllabique de ces emprunts sont pour beaucoup dans l'attribution du genre. L'étude du corpus a révélé que le phonème " $\mathrm{a}$ » et la syllabation (syllabe ouverte CV ou fermée CVC) ont une incidence majeure sur la determination du genre comme je vais le démontrer plus bas.

\section{1- La conservation du genre d'origine}

Comme nous l'avons signalé plus haut, certains emprunts au français ont pu garder leur genre d'origine. Dans les paragraphes suivants, nous allons donner les différents cas de conservation du genre et essayer de trouver les raisons morphosyntaxiques $d u$ comportement de ce type d'emprunts.

\subsection{1- Du féminin français au féminin marocain}

27 Il entre dans cette catégorie les substantifs français féminins qui se terminent par des syllabes fermées de type CVC à l'oral, mots terminés par un «e » muet graphique. Celles-ci prennent la marque du féminin marocain /a/

$$
\begin{aligned}
& \text { Une pelle } \rightarrow[\text { bala }] \\
& \text { Une brouette } \rightarrow\left[\text { barwit }^{\mathrm{f}} a\right] \\
& \text { Une patate } \rightarrow\left[\text { bat }^{\mathrm{f}} a t^{\mathrm{f}} \mathrm{a}\right] \\
& \text { Une baraque } \rightarrow\left[\text { bərraka }^{2}\right.
\end{aligned}
$$


minisation de ces emprunts ne déroge pas à la règle générale du féminin en arabe marocain qui consiste à suffixer un /-a/ aux mots masculins dépourvus de terminaison (Iraqui Sinaceur, 2004, p. 417). Il convient de souligner également que cette manière d'obtenir le féminin est héritée de l'arabe standard qui marque le féminin, en règle générale, par l'ajout du suffixe /-a/ comme le montrent les exemples suivants :

/mudarris/ (un enseignant) $\rightarrow$ /mudarrisa/ (une enseignante)

$/ \mathrm{katib} /$ (un écrivain) $\rightarrow /$ katiba/ (une écrivaine)

\subsection{2- Du masculin français au masculin marocain}

À cette catégorie appartiennent les lexies masculines qui n'ont pas changé de genre lorsqu'elles ont été transférées à l'arabe marocain, c'est-à-dire, elles ont le même genre (masculin) dans les deux systèmes linguistiques français et marocain.

$$
\begin{aligned}
& \text { Un virage } \rightarrow \text { [vira3] } \\
& \text { Un téléphone } \rightarrow \text { [tilifun }] \\
& \text { Un tableau } \rightarrow\left[\mathrm{t}^{\text {`ablo }}\right]
\end{aligned}
$$

Les exemples ci-dessus montrent que cette catégorie de vocables comprend aussi bien les mots à syllabes fermées comme [tilifu:n] que ceux à syllabes ouvertes tel [tªblo].

\section{2- Acquisition du genre de la langue emprunteuse}

Dans l'étude du corpus, il s'est avéré qu'il y a eu beaucoup d'emprunts qui ont changé leur genre d'origine et acquis celui de la langue emprunteuse. Dans les lignes qui suivent, nous examinerons ce genre d'emprunts cas par cas.

\subsection{1- Du féminin au masculin}

Après l'analyse des emprunts, nous avons tiré deux conclusions concernant cette catégorie de mots.

- Tous les substantifs féminins français qui se terminent par une syllabe fermée avec un $e$ muet à la fin et n'ont pas reçu la marque du féminin marocain /a/ s'approprient le genre masculin marocain.

Féminin $\rightarrow$ masculin

Moquette $\rightarrow$ [muket]

Godasse $\rightarrow$ [gud'as]

Confiture $\rightarrow$ [kufitir]

Sacoche $\rightarrow$ [ akuf]

- Sont masculinisés aussi les mots français se terminant par la voyelle nasale [jõ] (réalisée [ju:n] $)^{5}$ en arabe marocain.

Féminin $\rightarrow$ masculin

Télévision $\rightarrow$ [tilivizju:n]

Permission $\rightarrow$ [bərməsju:n]

La masculinisation de ce type de mots peut être attribuée à l'influence de l'arabe classique sur l'arabe marocain au fil du temps, cette modification du genre est due au fait que les mots que l'arabe marocain a hérités de l'arabe classique, ayant en position finale [u:n], sont au masculin.

Exemple : [qanu:n] (une loi), [maznu:n] (fou), [mad'mu:n] (contenu) 

la morphosyntaxe marocaine, ils n'échappent pas aux règles de l'arabe marocain en la matière. La majorité des emprunts français ont un pluriel régulier. Cela veut dire que le pluriel est marqué par l'ajout de suffixes spécifiques. Ces suffixes ne sont pas systématiques, ils dépendent de la structure phonique des emprunts. Certains reçoivent des désinences en fin de mot, d'autres subissent des modifications internes. 


\section{1- Le nombre pluriel}

39 À l'instar de l'arabe classique, il y a deux manières de construire le pluriel en arabe marocain (Iraqui Sinaceur, 2004, p. 418) :

- L'adjonction d'un suffixe à la fin du mot, c'est le pluriel dit externe.

- Une modification complète du schème, c'est le pluriel interne (pluriel brisé)

\subsection{1- Le pluriel externe :} celui-ci. Il s'agit de la désinence /a:t/(Iraqui Sinaceur, 2004, p. 419). Cette forme n'est pas la seule, mais elle est la plus répandue et la plus productive parce qu'elle est inspirée directement de l'arabe classique dont la plupart des mots pluriels est obtenu en ajoutant la marque /a:t/.

Singulier $\rightarrow$ pluriel

[Hammam] « un bain » $\rightarrow$ [Hammama:t] « des bains »

[maktaba] « une librairie » $\rightarrow[$ maktaba:t] « des librairies »

Les emprunts au français, comme on le verra plus loin, peuvent avoir d'autres formes de pluriel. Ces formes plurielles sont obtenues en ajoutant les suffixes suivants : /i:n/ et /a:t/ (Iraqui Sinaceur, 2004, p. 419).

\section{a) Pluriel externe obtenu par adjonction de /a:t/}

- Les mots à syllabe fermée :

Considérons les exemples suivants :

Singulier $\rightarrow$ pluriel

$[\mathrm{kabl}]$ « un câble » $\rightarrow[$ kabla:t $]$ « des câbles »

[karja:n] « une carrière » $\rightarrow[$ karjana:t] « des carrières »

De ces exemples et de ceux qui suivent, on constate une grande différence entre la formation du pluriel des mots arabes eux-mêmes et celle des emprunts au français. Pour le pluriel des mots arabes eux-mêmes, le genre du mot est déterminant dans le choix de la marque à mettre à la fin, ainsi on ne retrouve la terminaison /a:t/ qu'à la fin des mots arabes féminins. Alors que ce critère du genre du mot n'est pas pris en compte pour les emprunts au français, le mot $/ \mathrm{kabl} /$, par exemple, est un substantif masculin, pourtant il porte la marque /a:t/ au pluriel.

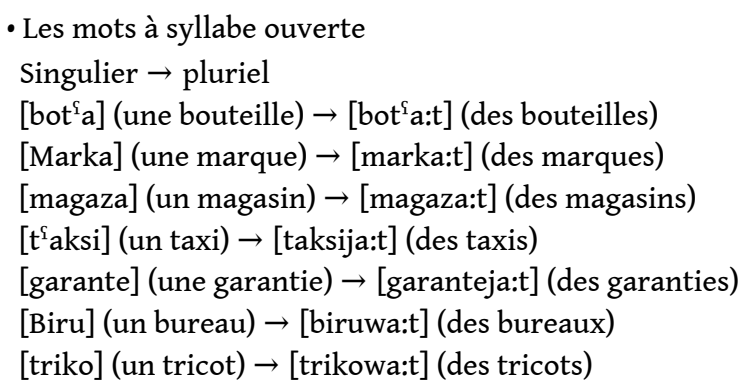

Les exemples ci-dessus montrent que les mots empruntés féminins en arabe marocain marqués par la voyelle [a] au singulier perdent cette inflexion au profit du pluriel /a:t/. En revanche, les autres mots (masculins) avec les voyelles [e] et [i] à la fin prennent un yod, tandis que ceux avec les voyelles [o] et [u] à la fin reçoivent la semi-consonne [w]. Ces éléments phoniques sont ajoutés aux mots pour rendre la prononciation plus fluide. 
45 Le jod [j] peut, dans certains cas, remplacer [w]. Comme dans les deux exemples suivants :

[biru] (un bureau) $\rightarrow$ [biruja:t] (des bureaux) (au lieu de biruwa:t)

[zero] (un zéro) $\rightarrow$ [zeruj:at] (des zéros) (au lieu de ziruwa:t)

\section{b) Pluriel externe obtenu par l'adjonction de [i:n]}

46 Au même titre qu'en arabe standard, où le pluriel masculin des noms (ainsi que des adjectifs) peut être formé en ajoutant /i :n/ (ou /u:n/ selon la syntaxique de ces mots dans la phrase) ${ }^{6}$. En arabe marocain, le pluriel des adjectifs empruntés au français est formé en ajoutant à la fin la terminaison du pluriel /i:n/ sans aucune modification. À l'exception d'un yod / $\mathrm{j} /$ qui est inséré juste avant la marque du pluriel pour faciliter la prononciation.

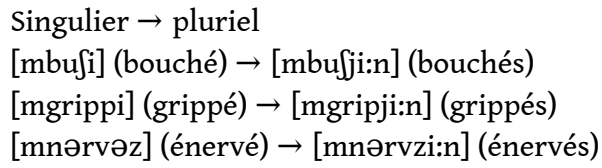

Notez que l'ajout du yod /j/ est propre aux adjectifs avec la voyelle [i] à la fin, comme le montrent les exemples ci-dessus.

\section{c) Pluriel externe obtenu par adjonction de [a]}

Ce suffixe est moins productif, car il est principalement utilisé pour les noms désignant des activités professionnelles ou habituelles. Cette construction est issue de la forme plurielle de certains mots d'origine arabe.

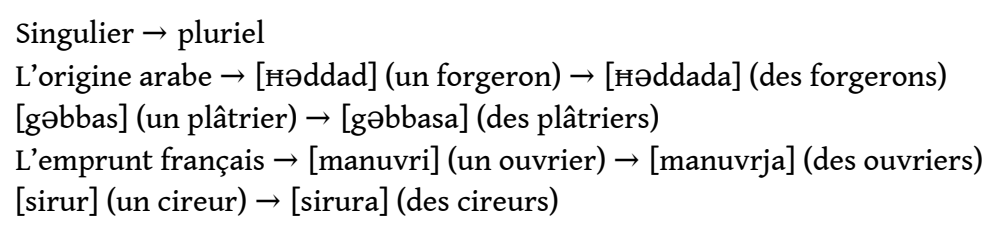

\subsection{2- Le pluriel interne (brisé)}

Ce sont les mots qui subissent une modification interne au pluriel (une modification complète du schème) tout en conservant la racine du mot singulier, notamment les mots à racine bilitère et ceux à racine trilitère. Du point de vue lexical, il s'agit de la formation d'un nouveau lexème. Ce genre de pluriel concerne aussi bien les substantifs que les adjectifs.

Après examen du corpus nous avons relevé trois manières de constituer ce genre de pluriel en fonction du nombre de consonnes des mots au singulier.

\section{a) Le pluriel des mots à racine bilitère}

51 Dans ce paragraphe nous allons donner le pluriel des différentes formations des mots à racine bilitère :

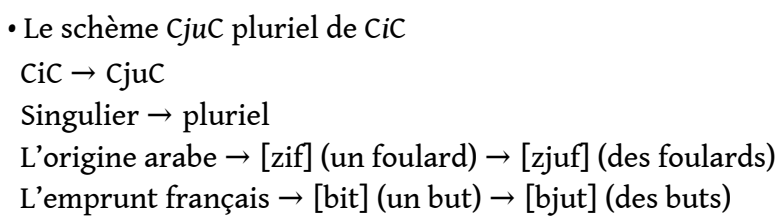


Cette structure trouve son origine dans l'arabe standard dont le pluriel fucul est forgé à partir de la forme singulière facl comme dans l'exemple suivant :

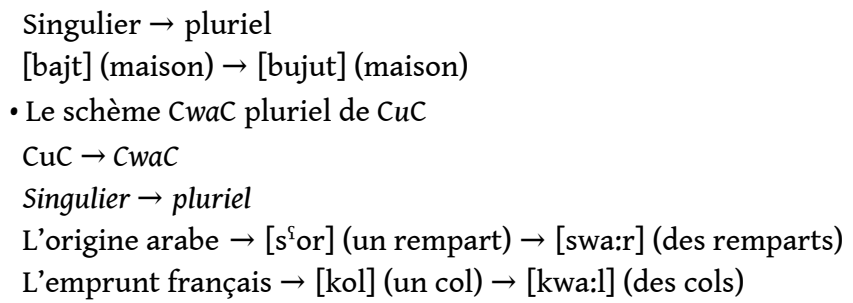

Cette forme de pluriel trouve son origine en arabe classique comme il est montré dans l'exemple ci-après :

Singulier $\rightarrow$ pluriel

[nu:r] (une lumière) $\rightarrow$ [anwa:r] (des lumières)

La chute du hamza de afEa:l a donné lieu au schème marocain $f \varepsilon a: l$.

- Le schème CiCa:n pluriel de $\mathrm{Ca}: \mathrm{C}$

Ca: $\mathrm{C} \rightarrow$ CiCa:n

Ce cas concerne surtout les mots singuliers monosyllabiques du type Ca:C. (Iraqui Sinaceur, 2004, p. 419) ces derniers subissent au pluriel les transformations suivantes :

- le remplacement de [a] par [i].

- l'adjonction de la désinence [a:n] à la fin du mot au pluriel.

Exemples :

Singulier $\rightarrow$ pluriel

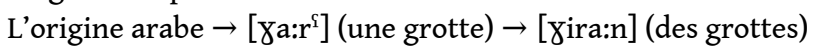

$\left[\right.$ fa: $\left.r^{\mathrm{I}}\right]$ (une souris) $\rightarrow[$ fira:n] (des souris)

L'emprunt français $\rightarrow$ [ka:ri $]$ (un car) $\rightarrow\left[\right.$ kir $\left.^{\mathrm{f}} \mathrm{a}: \mathrm{n}\right]$ (des cars)

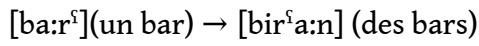

\section{b) Le pluriel des mots à racine trilitère}

57 Le pluriel des emprunts à racine trilitère sont construit de la même manière que leurs équivalents marocains ; c'est à dire qu'il y a une adaptation au niveau de la formation du pluriel. Nous allons présenter dans cette section les différentes constructions de pluriel pour cette catégorie de mots (à racine trilitère).

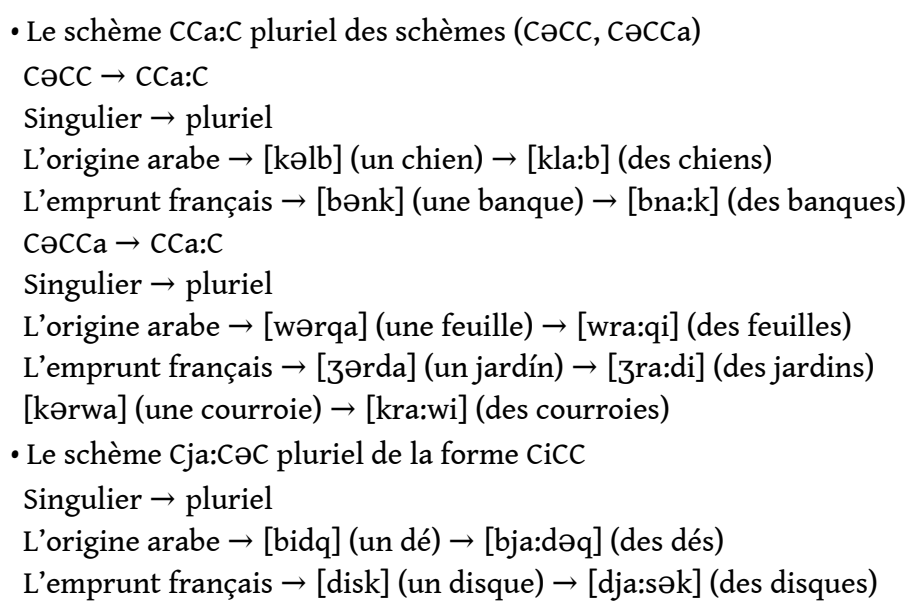

En arabe marocain, le schème de ce pluriel ( $\mathrm{Cja}: \mathrm{C} \partial \mathrm{C})$ est construit sur le schème singulier (Ci:Cu:C) : [zi:zo:n] (un sourd-muet) $\rightarrow$ [zja:zən] (des sourds-muets). 
Ce qui veut dire que le pluriel des emprunts (CiCC) n'est pas d'origine arabe.

- Le schème $\mathrm{C} w a: \mathrm{C} \partial \mathrm{C}$ pluriel de $\mathrm{CaCu}: \mathrm{C}$ (masculin) et de $\mathrm{CaCiCa}$ (féminin)

$\mathrm{CaCu}: \mathrm{C} \rightarrow \mathrm{Cwa}: \mathrm{CəC}$

Singulier $\rightarrow$ pluriel

L'origine arabe $\rightarrow$ [ríabu:z] (un soufflet) $\rightarrow\left[\mathrm{r}^{\mathrm{I}}\right.$ wa:bəz $]$ (des soufflets)

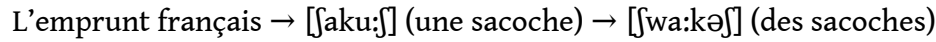

CaCiCa $\rightarrow$ Cwa:CoC

Singulier $\rightarrow$ pluriel

L'origine arabe $\rightarrow$ [qamiza] (une chemise) $\rightarrow$ [qwa:mə3] (des chemises)

L'emprunt français $\rightarrow$ [gamila] (une gamelle) $\rightarrow$ [gwa:məl] (des gamelles)

- Le schème CCa:jC pluriel de CCaCa

$\mathrm{CCaCa} \rightarrow \mathrm{CCa}$ :jC

Singulier $\rightarrow$ Pluriel

L'origine arabe $\rightarrow\left[b^{\mathrm{f}}\right.$ ana] (une peau de mouton) [bt $\mathrm{f}^{\mathrm{f}} \mathrm{a}$ :jn] (des peaux de mouton)

L'emprunt français $\rightarrow\left[\mathrm{bl}^{\mathrm{I}} \mathrm{a}: \mathrm{sa}\right]$ (une place) [bl $\left.{ }^{\mathrm{I}} \mathrm{a}: \mathrm{js}\right]$ (des places)

Le pluriel interne dans ce dernier cas entraine la perte de la marque du féminin /a/.

- Le schème CCa:Ci pluriel de CəCCa

CәCC $a=>\mathrm{CCa}: \mathrm{Ci}$

61 Ce sont des mots trilitères qui prennent la marque /a/ au féminin. Au pluriel, ces vocables subissent deux sortes de changements :

- Interne : il s'agit de l'union des deux premières consonnes à l'initial du mot suivi de la voyelle longue /a:/

- Externe : le remplacement de la voyelle [a] du singulier par la voyelle [i] au pluriel

Singulier $\rightarrow$ Pluriel

L'origine arabe $\rightarrow$ [zərda] (un festin) $\rightarrow$ [zra:di] (des festins)

L'emprunt français $\rightarrow$ [mərka] (une marque) $\rightarrow$ [mra:ki] (des marques)

- Le schème $\mathrm{Cwa}: \mathrm{C} \partial \mathrm{C}$ pluriel de $\mathrm{Ca}: \mathrm{CC} a$

\section{$\mathrm{Ca}: \mathrm{CCa} \rightarrow \mathrm{Cwa}: \mathrm{CoC}$}

Singulier $\rightarrow$ pluriel

L'origine arabe $\rightarrow\left[\right.$ Jant $\left.{ }^{\uparrow} a\right]$ (une valise) $\rightarrow\left[\right.$ [wa:nət $\left.{ }^{\uparrow}\right]$ (des valises)

L'emprunt français $\rightarrow$ [sagma] (un segment) $\rightarrow$ [swa:gəm] (des segments)

- Le schème $\mathrm{CC}_{2} u: C_{2}$ pluriel de $\mathrm{C}_{2} \mathrm{C}_{2} \mathrm{C}_{2} a$

$\operatorname{CaC}_{2} \mathrm{C}_{2} \mathrm{a}=>\mathrm{CC}_{2} \mathbf{u}: \mathrm{C}_{2}$

Singulier $\rightarrow$ Pluriel

L'origine arabe $\rightarrow$ [qətta] (un chat) $\rightarrow$ [qtu:t] (des chats)

L'emprunt français $\rightarrow$ [qəffa] (un couffin) $\rightarrow$ [qfu:f] (des couffins)

\section{c) Le pluriel des mots à racine quadrilitère}

Ce sont les mots qui disposent dans leurs structures radicales de quatre consonnes au singulier, ces unités lexicales font leur pluriel par l'introduction au milieu de la voyelle longue [a:]. Le schème donc du pluriel sera $\mathrm{CCa}: \mathrm{C} \mathrm{C}$, plusieurs formes du singulier donnent ce genre de pluriel.

СәсСәC $\rightarrow$ cCa:CәC

Singulier $\rightarrow$ Pluriel

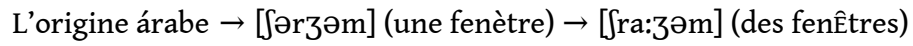

L'emprunt français $\rightarrow$ [tənbər] (un timbre) $\rightarrow$ [tna:bər] (des timbres)

$\mathrm{CaCCuCa} \rightarrow \mathrm{CCa}: \mathrm{CoC}$

Singulier $\rightarrow$ Pluriel

L'origine arabe $\rightarrow$ [xenfura] (un museau) $\rightarrow$ [xna:fər] (des museaux)

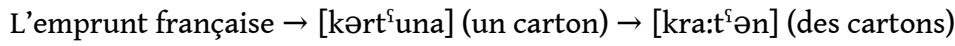

CәCCa:Ca $\rightarrow$ CCa:CәC 
Singulier $\rightarrow$ Pluriel

L'origine arabe $\rightarrow$ [ əkka:ra] (un cartable) [Ska:kər] (des cartables)

L'emprunt français $\rightarrow$ [bərra:ka] (une baraque) [bra:rək] (des baraques) morphosyntaxiques que subissent les lexèmes français au cours de leur intégration dans l'arabe marocain, à savoir, l'attribution du genre et du nombre. 
71 Au niveau du genre, nous avons trouvé qu'il y a deux possibilités d'adaptation des emprunts au français à l'arabe marocain : soit ils conservent leur genre d'origine, soit ils acquièrent un genre nouveau. À partir des mots du corpus, nous avons tenté d'identifier les caractéristiques communes des emprunts des deux catégories afin de mieux comprendre les raisons du maintien ou du changement de genre. À cet égard, il a été constaté que le plus grand nombre d'emprunts au français sont du genre féminin en arabe marocain, pour des raisons essentiellement phonétiques, notamment phonétiques articulatoires. Ceci est dû au fait que les locutrices et les locuteurs ont souvent tendance à remplacer les unités phonétiques de la langue d'origine par leurs équivalents les plus proches dans la langue réceptrice.

Au niveau du nombre, nous avons trouvé que les mots d'origine française sont fortement influencés par la morphosyntaxe marocaine, la majorité des emprunts au français respectent les règles de l'arabe marocain. En effet, un grand nombre de ces emprunts, à l'instar de l'arabe, ont un pluriel régulier qui se construit par d'adjonction des suffixes spécifiques, toutefois certains autres ont un pluriel irrégulier obtenu par des modifications internes liées à la structure syllabique des mots.

\section{BIBLIOGRAPHIE}

BAGGIONI, D. (1994). «Schuchardt et mixité des langues », Travaux du Cercle Linguistique d'Aix-enProvence.

CHADELAT, J.-M. (2000). Valeur et fonctions des mots français en anglais à l'époque contemporaine. Paris : Éd. L'Harmattan.

DE GOURMONT, R. (1988). Esthétique de la langue française. Paris : Éd. Mercure de France.

DEROY, L. (1956). L'emprunt linguistique, Paris : Éd. Les belles lettres ».

DU BELLAY, J. (2013) Défense et illustration de la langue française. Paris : Presses électroniques de France.

DUBOIS, J. et al. (1973). Dictionnaire de linguistique. Paris : Larousse.

DUBOIS, J. et al. (1980). Dictionnaire de linguistique. Paris : Larousse.

EL GARNI, M. (2020). « Les emprunts lexicaux de l'arabe marocain au français : étude phonologique ». Synergies Chili, 16, p. 99-129.

ETIEMBLE, R. (1964). Parlez-vous franglais ?. Paris : Folio.

GUILBERT, L. (1975). La créativité lexicale. Paris : Larousse.

HAGEGE, C. (2006). Combat pour le français : Au nom de la diversité des langues et des cultures. Paris : O. Jacob.

HAMERS, J. F. (1997). «Emprunt ». Sociolinguistique. Concept de base. Bruxelles : Mardaga, p. 136-139.

HAUGEN, E. (1950). « The analysis of linguistic borrowing ». Language, 26 (1), p. 210-231. 
HUMBLEY, J. (1974). « Vers une typologie de l'emprunt linguistique ». Cahiers de lexicologie, 25 (2), p. 46-70.

IRAQUI SINACEUR, Z. (1994). Le Dictionnaire Colin d'arabe dialectal marocain. Rabat: Al Manahil, Ministère des affaires culturelles.

IRAQUI SINACEUR, Z. (2004). « Histoire et emprunt linguistique ». in : Dakhlia, J. Trames de langues : Usages et métissages linguistiques dans l'histoire du Maghreb. Tunis : Institut de recherche sur le Maghreb contemporain, p. 414-426.

LOUBIER, C. (2011). De l'usage de l'emprunt linguistique. Montréal : Office québécois de la langue française.

MEILLET, A. (1921). Linguistique historique et linguistique générale. Paris : É. Champion.

MGHERFAOUI, K. et al. (2017). Qamus ad-dariza almayribiya. Casablanca : Centre Zagora pour le Développement du dialecte.

MYERS-SCOTTON, C. (2002). Language contact: Bilingual encounters and grammatical outcomes. Oxford : Oxford University Press.

QUEMADA, B. (1971). « Banque de mots ». in : Sablayrolles J.-F., Les néologismes. Paris : Presses universitaires de France.

REY-DEBOVE, J. (1973). « La sémiotique de l'emprunt lexical ». Travaux de Linguistique et de Littérature, 11, p. 109-123.

SCHUCHARDT, H. (1925). Der Individualismus in der Sprachforschung, 202. Vienne : Hölder-PichlerTempsky.

TOURNIER, J. (1985). Introduction descriptive à la lexicogénétique de l'anglais contemporain. Paris/ Genève : H. Champion/slatkine.

\section{NOTES}

1. à l'image de H. Schuchardt (1925) qui pense que l'emprunt est « une réalité centrale de l'activité langagière.» (Schuchardt, 1925, cité par Baggioni, 1994, p. 3)

2. HAGEGE, C. (2006). Combat pour le français: Au nom de la diversité des langues et des cultures. O. Jacob. (p. 42)

3. Il convient de souligner que le terme emprunt peut être utilisé dans ces deux acceptions « action d'emprunter » et la « chose empruntée ». La majorité des linguistes utilisent l'emprunt linguistique dans son sens premier, c'est à dire l'action par laquelle une langue intègre un élément d'une autre langue. Seule une minorité de spécialistes l'envisagent dans le second sens (chose empruntée).

4. Deroy, L. (1956), L'Emprunt linguistique, Paris : Éd. Les belles lettres, p. 20

5. La dénasalisation est systématique en arabe marocain. Comme il n'existe pas de voyelles nasales en arabe marocain, il est évident que les locuteurs marocains procèdent à la dénasalisation de ces voyelles. Ainsi, ce type de voyelle est articulé par le locuteur monolingue comme une combinaison de deux sons (voyelle + consonne) ou comme une simple voyelle orale.

6. Par exemple, lorsque le nom (pluriel masculin) est sujet, il prend le suffixe /u:n/ et lorsqu'il est complément, il prend la déclinaison /i:n/. 


\section{RÉSUMÉS}

L'emprunt linguistique est considéré par les linguistes, à l'exception de quelques puristes, comme un mécanisme linguistique essentiel pour l'enrichissement des langues. Toutes les caractéristiques linguistiques (orthographe, phonologie, grammaire, lexique ...) de la langue peuvent être empruntées.

Pour qu'il soit complètement intégré par la langue d'accueil, l'emprunt lexical doit subir des modifications phonologiques et morphosyntaxiques. Dans cette étude, nous nous sommes centrés, à partir d'un corpus écrit et d'un corpus oral obtenu par enquête, sur un aspect morphosyntaxique spécifique de l'intégration des emprunts lexicaux au français, à savoir l'attribution du genre et du nombre à ces lexèmes d'origine française. La présente étude montre que le comportement des emprunts en termes de genre et de nombre n'est pas standardisé : certains gardent le genre d'origine, d'autres le perdent au profit de la langue emprunteuse. En ce qui concerne le nombre, nous avons trouvé que les emprunts ont pu intégrer les règles du nombre de l'arabe marocain. La description sera suivie d'une discussion sur les raisons linguistiques qui peuvent expliquer les différents comportements de ces emprunts en termes de genre et de nombre.

Language borrowing is considered by linguists, with the exception of a few purists, as an essential linguistic mechanism for language enrichment. All the linguistic characteristics (spelling, phonology, grammar, lexicon, etc.) of the language can be borrowed.

In order to be fully integrated by the host language, lexical borrowing must undergo phonological and morphosyntactic modifications. In this study, we have focused, on the basis of a written corpus and an oral corpus obtained by survey, on a specific morphosyntactic aspect of the integration of lexical borrowings from French, namely the attribution of gender and number to these lexemes of French origin. The present study shows that the behaviour of borrowings in terms of gender and number is not standardised: some keep the original gender, others lose it to the borrower's language. As far as numbers are concerned, we found that the borrowers may have incorporated the rules of Moroccan Arabic numbers. The description will be followed by a discussion of the linguistic reasons that may explain the different behaviour of these borrowers in terms of gender and number.

\section{INDEX}

Mots-clés : emprunt lexical, intégration, genre, nombre, arabe marocain, Français

Keywords : lexical borrowing, integration, gender, number, Moroccan Arabic, French 